\title{
Role of Sodium Channel on Cardiac Action Potential
}

\author{
S. H. Sabzpoushan \\ Department of Biomedical Engineering \\ Iran University of Science and Technology (IUST) \\ Tehran, Iran \\ sabzposh@iust.ac.ir
}

\author{
A. Faghani Ghodrat \\ Department of Biomedical Engineering \\ Iran University of Science and Technology (IUST) \\ Tehran, Iran \\ alireza.faghani.ghodrat@gmail.com
}

\begin{abstract}
Sudden cardiac death is a major cause of death worldwide. In most cases, it's caused by abnormal action potential propagation that leads to cardiac arrhythmia. The aim of this article is to study the abnormal action potential propagation through sodium ion concentration variations. We use a new electrophysiological model that is both detailed and computationally efficient. This efficient model is based on the partial differential equation method. The central finite difference method is used for numerical solving of the two-dimensional (2D) wave propagation equation. Simulations are implemented in two stages, as a single cardiac cell and as a two-dimensional grid of cells. In both stages, the normal action potential formation in case of a single cell and it's normal propagation in case of a twodimensional grid of cells were simulated with nominal sodium ion conductance. Then, the effect of sodium ion concentration on the action potential signal was studied by reducing the sodium ion conductance. It is concluded that reducing the sodium ion conductance, decreases both passing ability and conduction velocity of the action potential wave front.
\end{abstract}

Keywords: action potential; sodium channel; partial differential equation; arrhythmia; reentrant

\section{INTRODUCTION}

Information transmission in the form of propagating waves of electrical excitation is the fastest form of long-range internal communications available to living organisms. In the heart, the electrical waves initiate contractions of the cardiac muscle. Abnormal propagation of the electrical excitation wave has serious medical consequences. Regarding the brain, it is believed to be associated with epilepsy. Regarding the heart it leads to cardiac arrhythmia. Among the various types of cardiac arrhythmias, reentrant ventricular arrhythmia is considered the most dangerous. Reentrant arrhythmia which frequently occurs, is caused mainly due to abnormal reentrant propagation of excitation waves and is considered very dangerous too [1].

Modeling studies have a long-standing tradition and play a very significant role in cardiac electrophysiological research. Mathematical models and computer simulations play an increasingly important role in cardiac arrhythmia research. Ionic (electrophysiological) models include complete detailed descriptions of cardiac cells processes and can be used for accurate studies in cellular level. One of the most important application of these theoretical studies is the simulation of the human heart, which is rather important for a number of reasons. Firstly, the possibilities for doing experimental and clinical studies are very limited. Secondly, animal hearts used for experimental studies may differ significantly from the human heart in terms of size, heart rate, action potential (AP), shape, duration and restitution, vulnerability to arrhythmias, etc. Finally, cardiac arrhythmias, especially those occurring in the ventricles, are three-dimensional phenomena whereas experimental observations are still largely constrained to surface recordings. Some of the above mentioned problems can be bypassed using computer simulations of arrhythmias in the human heart [1-2].

The propagation of a wave front in an excitable media requires a sufficient number of $\mathrm{Na}$ ions that can diffuse into adjacent resting cells and raise the membrane potential to the firing threshold. Na channels become rapidly inactivate, so they participate only briefly in the wave front expansion and therefore must be replaced with newly recruited channels in order to keep the wave front from collapsing. The process of charge diffusion, subsequent regional depolarization and opening of $\mathrm{Na}$ channels characterizes the recruiting process [3]. The recruiting capacity reflects the density of non-inactivated and unblocked $\mathrm{Na}$ channels adjoining the leading edge of the wave front. The status of an impulse arising from stimulation is thus determined by the ability of the wave front to recruit sufficient $\mathrm{Na}$ channels from adjacent cells such that the resulting inward current maintains an active region comparable in size to the liminal length.

The inward $\mathrm{Na}$ current $(\mathrm{INa})$ is responsible for the initial upstroke of the action potential. Equations for INa are of the type first formulated by Beeler and Reuter [4]. The steady-state activation and inactivation curves used in the model are based on recent patch clamp experiments in rat ventricular myocytes [5]. The basic kinetic characteristics of INa are similar in ventricular cells across different species [6]. Therefore, the time constants for activation and inactivation were adapted from the guinea pig ventricular cell model [7], and were scaled for room temperature [8]. The maximum Na conductance $\left(\mathrm{G}_{\mathrm{Na}}\right)$ was adjusted to generate an appropriate value for the action potential amplitude and the maximal upstroke velocity $\left(\mathrm{dV} / \mathrm{dt}_{\max }\right)$. Recent clinical reports of gene mutations, drugs and cardiac disease link a variety of often lethal conditions with the loss of cardiac $\mathrm{Na}$ channel function (NaLOF) and reentrant proarrhythmia. 


\section{METHOD}

A human cardiac cell model that is both detailed and computationally efficient is needed in order to study human heart activities and the way these activities are linked to (sub)cellular processes such as ion channel mutations. In this work, we use a new electrophysiological model that is both detailed and computationally efficient [2]. The action potential generation and propagation was described using the following differential equation [9]:

$$
C_{m} \frac{\partial V}{\partial t}=I_{i o n}+I_{s t i m}+D \nabla V
$$

where $\mathrm{V}$ is the transmembrane potential, $\mathrm{C}_{\mathrm{m}}$ is the membrane capacitance, $\mathrm{D}$ is the diffusion coefficient, $\mathrm{I}_{\text {ion }}$ denotes sum of the currents constituted by various ions across the cell membrane and $I_{\text {stim }}$ is the external current signal. The nominal value of sodium ion conductance is $15 \mathrm{mS} / \mathrm{cm}^{2}$. For the numerical solving of the two-dimensional (2D) wave propagation, the central finite difference method [10] is used with a time step of 0.02 millisecond and a space step of 0.25 millimeter. We assume $\mathrm{C}=1 \mu \mathrm{F} / \mathrm{cm}^{2}, \mathrm{D}=0.00154 \mathrm{~cm}^{2} / \mathrm{ms}$ in two-dimensional grid of cells simulations to obtain a maximum planar conduction velocity $(\mathrm{CV})$ of $68 \mathrm{~cm} / \mathrm{s}$ consistent with measurements in human ventricular tissue [2], and $\mathrm{D}=0 \mathrm{~cm}^{2} / \mathrm{ms}$ for single cells.

Simulations of cardiac action potential are implemented in two stages, as a single cardiac cell and as a two-dimensional grid of cells. Normal action potential formation in case of a single cell and it's normal propagation in case of a twodimensional grid of cells are simulated with nominal sodium ion conductance. The effect of sodium ion concentration on the action potential is studied by reducing the sodium ion conductance. Computer simulations are implemented using DELPHI .

\section{RESULTS}

The inward $\mathrm{Na}$ current (INa) is responsible for the upstroke of the action potential signal. In this section, we initially show the effect of sodium ion conductance on the action potential amplitude in case of a single cell and we investigate this effect on both amplitude and conduction velocity $(\mathrm{CV})$ of the action potential wave in a two-dimensional grid of cells.

\section{A. Single Cell}

The single cell model was constructed and simulated according to Tusscher's myocardial cell model [2]. The typical cardiac action potential has 5 phases from 0 up to 4 including: (0) depolarization or upstroke phase, (1) partial repolarization or notch phase (2) plateau phase (3) repolarization phase and (4) resting membrane potential phase. In mammalian heart, depolarization phase (Phase 0) results from a rapid inward pulse of sodium ions to a positive potential. In this section, the action potential response of a single cell is simulated with the nominal value of $\mathrm{G}_{\mathrm{Na}}$, as shown in Figure 1. Five phases for the action potential signal are observable. The vertical axis shows the transmembrane voltage in millivolts $(\mathrm{mV})$ and the horizontal axis shows the time in milliseconds (ms). At the next step, we decrease the $\mathrm{G}_{\mathrm{Na}}$ from its nominal value. Figures 2 and 3 show that when $\mathrm{G}_{\mathrm{Na}}$ was reduced to 70 and 50 percent of its nominal value, the action potential upstroke was decreased from $38 \mathrm{mV}$ to $30 \mathrm{mV}$ and $25 \mathrm{mV}$ respectively.

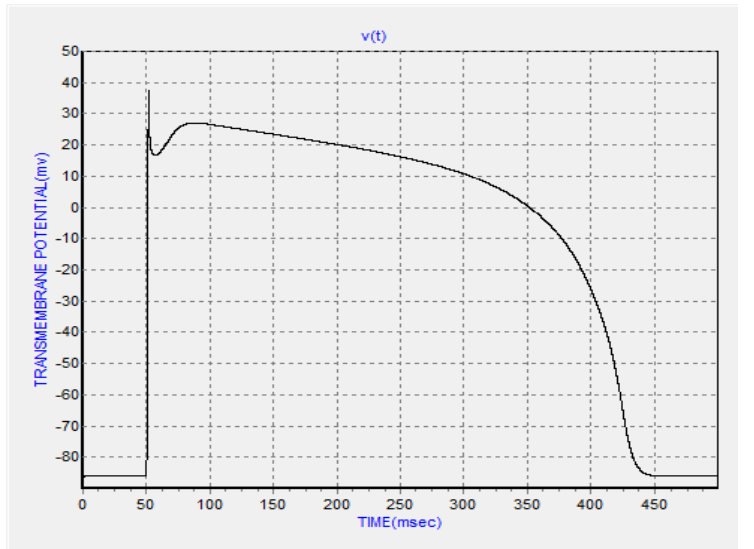

Fig. 1. Action potential of the single cell with the nominal value of $\mathrm{G}_{\mathrm{Na}}$

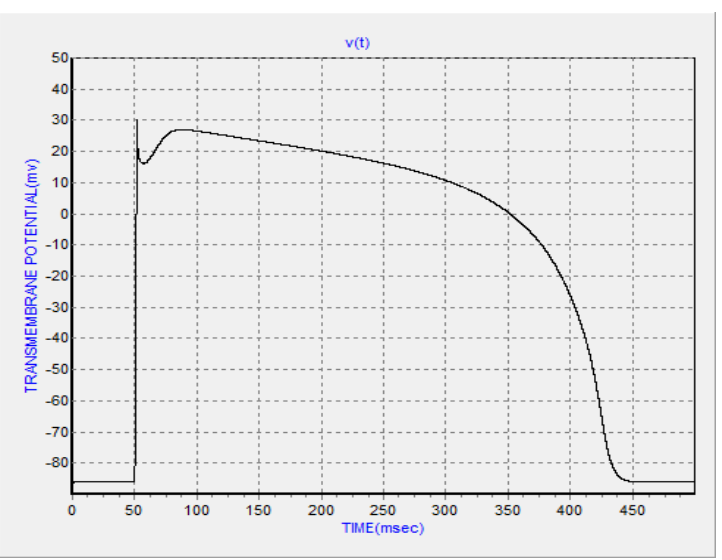

Fig. 2. Action potential of the single cell after decreasing $\mathrm{G}_{\mathrm{Na}}$ to $70 \%$ of its nominal value

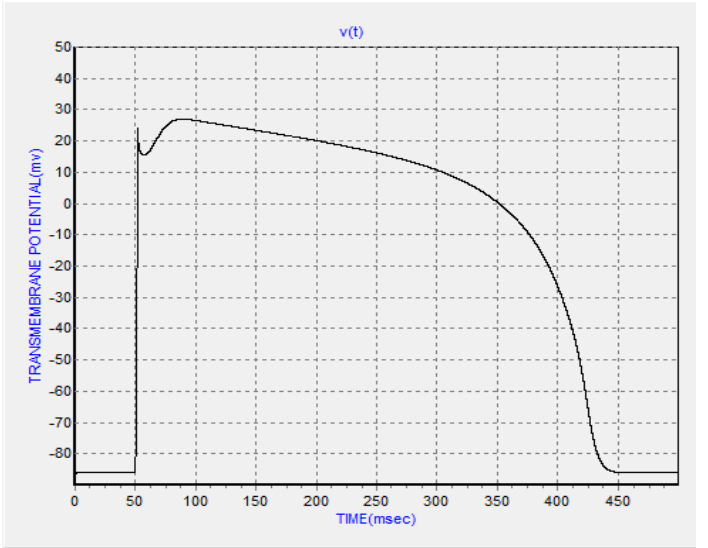

Fig. 3. Action potential of the single cell after decreasing $\mathrm{G}_{\mathrm{Na}}$ to $50 \%$ of its nominal value 


\section{B. Two-Dimensional Grid of Cells}

The two dimensional (2D) grid is a MxM grid of cells, where $\mathrm{M}$ is the number of cells. We test the $2 \mathrm{D}$ action potential propagation with different sodium ion conductances and show the effect of this operation on action potential propagation. The nature of grid connection are based on the physiological conditions, i.e. in reality, cardiac cells communicate with the maximum possible number of connected neighbors. The simulations are performed in a square excitable medium with 80 cells in both $\mathrm{x}$ and $\mathrm{y}$ direction. At first, a $15 \mathrm{mS} / \mathrm{cm}^{2}$ conductance is employed and parameters are kept at the nominal value. An external stimulus is applied at the left down corner of our excitable medium. This external stimulus current $(\mathrm{eS} 1)$ is required in order to generate an action potential. The normal action potential propagates in the medium from the left down corner to the right top corner. The snapshots of normal action potential propagation are shown in Figure 4.
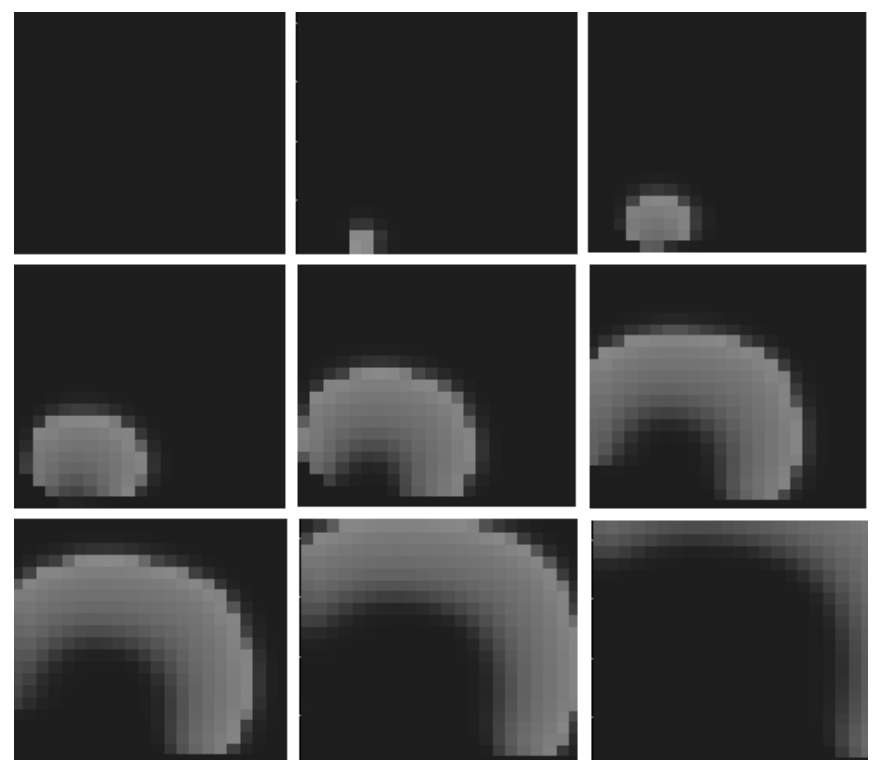

Fig. 4. $2 \mathrm{D}$ action potential propagation with nominal value of $\mathrm{G}_{\mathrm{Na}}$ (series of snapshots $0,0.5,2,4,6,8,10,12,14$ milliseconds after the start of propagation, from top row to bottom row respectively and in each row from left side to right side)

In the next study, the sodium ion conductance is decreased to 70 percent of its nominal value. The amplitude of the action potential wave front has decreased consequently, after the passing ability of the wave front has been reduced. Moreover according to the snapshots represented in Figure 5, the propagating wave front needs more time compared to the previous state to traverse across the medium whereas the conduction velocity of the wave front is reduced.

In the final state, we decrease the sodium ion conductance to 50 percent of its nominal value. Figure 6 depicts that both amplitude and conduction velocity of the action potential wave front has been further decreased compared to the two previous states. In figures 4-6, the dark color shows the medium resting state and the light color represents the medium depolarized state.
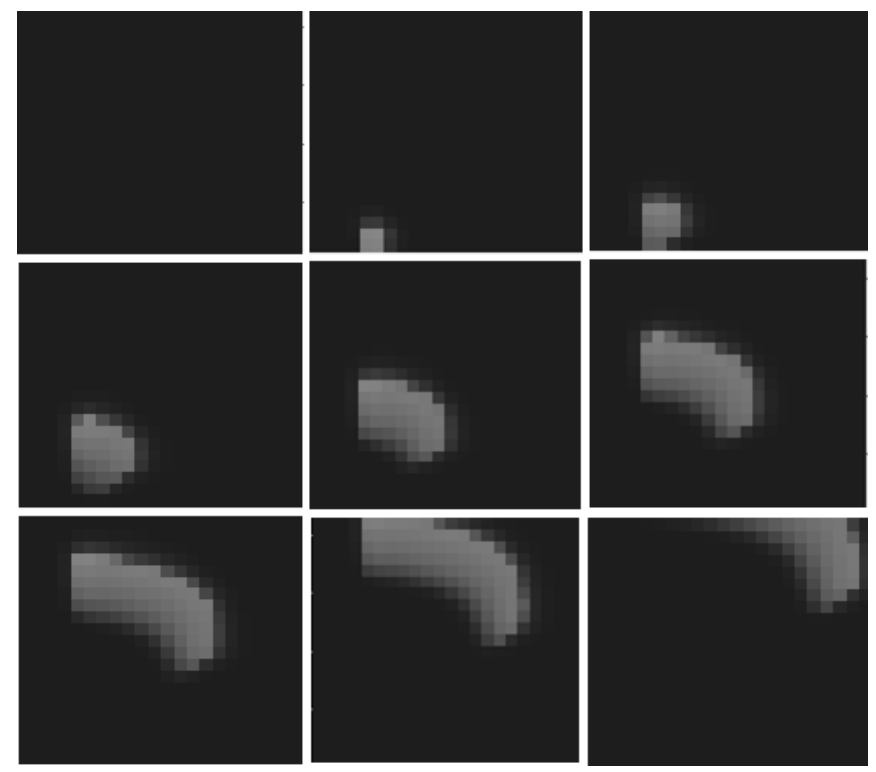

Fig. 5. 2D action potential propagation after decreasing $\mathrm{G}_{\mathrm{Na}}$ to $70 \%$ of its nominal value(series of snapshots $0,0.5,2,5,7,9,12,14,16$ milliseconds after the start of propagation, from top row to bottom row respectively and in each row from left side to right side)
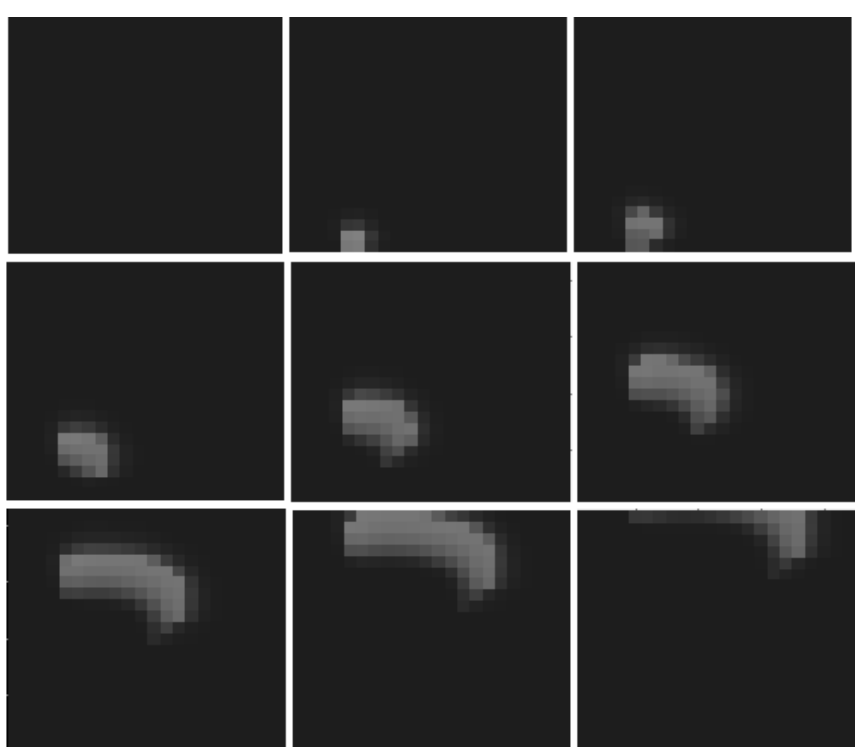

Fig. 6. 2D action potential propagation after decreasing $\mathrm{G}_{\mathrm{Na}}$ to $50 \%$ of its nominal value (series of snapshots $0,0.5,2,4,7,10,13,16,18$ milliseconds after the start of propagation, from top row to bottom row respectively and in each row from left side to right side)

As a next step, we study the effect of obstacles on the action potential propagation in our represented excitable medium. In the normal heart, rhythmic cardiac contractions are coordinated through nonlinear electrical waves of excitation that smoothly propagate through the cardiac tissue. Reentrant waves are a common cause of cardiac arrhythmias. The term re-entry was coined to describe a wave front that re-enters and hence re-excites the same tissue again and again as opposed to the normal planar wave front emitted by the sinus node that 
excites all tissue only once. In the two dimension tissue, reentry can be caused by a wave front curved around an obstacle [11-13]. The dead regions of the cardiac tissue play the role of obstacles.

In this study we create some local obstacles as dead tissues considering the sodium ion conductance equal to zero in the excitable medium. As shown in Figure 7, three rectangular obstacles in the medium are considered. The considered obstacles that exist in the passing way of action potential wave disorder wave front. This perturbation causes changes in the normal action potential propagation in the grid, which disturbs the normal cardiac rhythmic pattern and arrhythmia status occurs. The cumulative effect of the disturbance in the abnormal cardiac rhythmic pattern forms a re-entry in the location of the obstacle.

Re-entry is formed because the dead region of the tissue acts as an obstacle for the normal action potential propagation. As time elapses, the re-entry waves move to all regions of the grid and thus cause a total arrhythmic pattern. In Figures 4-7, it is clearly observed that sodium ion concentration plays a vital role in the propagation of the action potential wave front in an excitable medium.
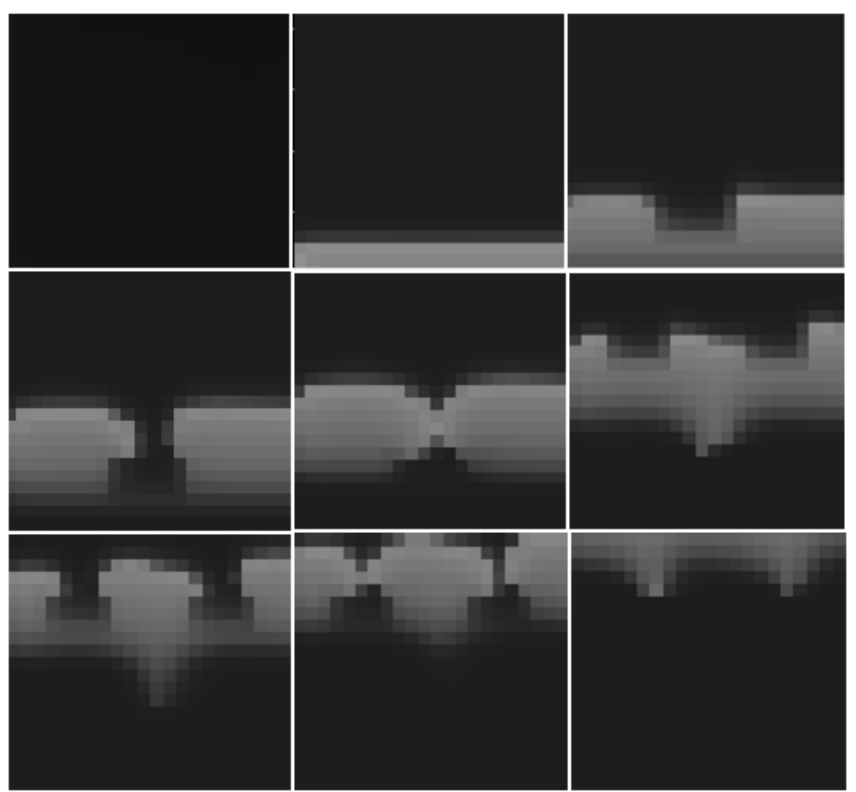

Fig. 7. Considering $\mathrm{G}_{\mathrm{Na}}$ equal to zero for creating obstacles which cause anatomical reentrant and consequently cardiac arrhythmia

\section{DISCUSSION}

In order for a wave front to successfully expand in tissue, recruitment of an adequate supply of charge carriers (e.g. Na) is required. The carriers should be able to diffuse down a concentration and voltage gradient into adjacent resting cells, depolarizing the local membrane potential to the Na channel opening threshold. Since recently recruited $\mathrm{Na}$ channels inactivate, they must be rapidly replaced with newly recruited channels in order to prevent wave front collapse. The process of charge diffusion and subsequent opening of resting $\mathrm{Na}$ channels adjacent to the wave front is referred as a recruiting process $[3,14]$. The amount of recruited charge, as determined by the recruiting range and the restored $\mathrm{Na}$ conductance density within this range, are critical determinants of not only whether propagation can be sustained but also of the propagation velocity. In this work, an efficient PDE model was used to study the action potential formation and its propagation. The effect of Sodium ion concentration, which is one of the reasons behind arrhythmias, was well studied in case of a single cell and in case of a 2D grid of cells. This was achieved by decreasing the sodium ion conductance step by step. The effects of the change were studied in each stage. In the final stage, we investigated the effect of obstacles on the action potential propagation. We considered three obstacles in the different points of the represented excitable medium by assigning 0 to $\mathrm{G}_{\mathrm{Na}}$. The obstacles played the role of dead regions of cardiac tissue and caused reentrant waves formation leading to cardiac arrhythmia

\section{CONCLUSION}

Results portrayed in this paper, confirmed that action potential propagation in an excitable media requires a sufficient number of $\mathrm{Na}$ ions that can diffuse into adjacent resting cells and raise the membrane potential to the firing threshold. It is concluded that decreasing the sodium ion conductance decreases both the passing ability and the conduction velocity of the action potential wave front. Sodium ion concentration plays a vital role in controlling arrhythmia, hence further research should be focused on studying the effects of this channel on formation and control of cardiac arrhythmias.

\section{REFERENCES}

[1] K. H. W. J. Ten Tusscher, "Spiral wave dynamics and ventricular arrhythmias", PhD Thesis, University of Utrecht, 2004.

[2] K. H. W. J. Ten Tusscher, A. V. Panfilov, "Cell model for efficient simulation of wave propagation in human ventricular tissue under normal and pathological conditions", Phys. Med. Biol., Vol. 51, pp. 6141-6156, 2006.

[3] C. Frank Starmer , A. O. Grant, T. J. Colatsky. "What happens when cardiac Na channel function is compromised? 2-Numerical studies of the vulnerable period in tissue altered by drugs", Cardiovascular Research, Vol. 57, No. 4, pp. 1062-1071, 2003.

[4] G. W. Beeler, H. Reuter, "Reconstruction of the action potential of ventricular myocardial fibres”, J. Physiol., Vol. 268, No. 1, pp. 177-210, 1977.

[5] H. C. Lee, T. Lu, N. L. Weintraub, M. VanRollins, A. A. Spector, E. F. Shibata. "Effects of epoxyeicosatrienoic acids on the cardiac sodium channels in isolated rat ventricular myocytes", J. Physiol., Vol. 519, pp. 153-168, 1999.

[6] D. A. Hanck, "Biophysics of sodium channels. In Cardiac Electrophysiology: From Cell to Bedside", D. P. Zipes and J. Jalife, editors. W. B. Saunders Co., Philadelphia, pp. 65-74, 1995.

[7] C. Luo, Y. Rudy, "A dynamic model of the cardiac ventricular action potential I simulations of ionic currents and concentration changes", Circ. Res., Vol. 74, pp. 1071-1096, 1994.

[8] T. J. Colatsky, "Voltage clamp measurements of sodium channel properties in rabbit cardiac Purkinje fibres", J. Physiol., Vol. 305, pp. 215-234, 1980.

[9] J. Keener, J. Sneyd, "Mathematical Physiology”, Springer, 1998.

[10] G. C. Everstine, “Numerical Solution of Partial Differential Equations”, Lecture Notes, George Washington University, 2010. 
[11] N. Wiener, A. Rosenblueth, "The mathematical formulation of the problem of conduction of impulses in a network of connected excitable elements, specifically in cardiac muscle". Arch. Inst. Cardiol. Mex, Vol. 16, No. 3, pp. 205-265, 1946.

[12] A. T. Winfree, "The geometry of biological time". Springer-Verlag, New York, USA, 1980.

[13] V. S. Zykov, "Simulation of wave processes in Excitable Media". Manchester University Press, Manchester, UK, 1987.

[14] C. F. Starmer, T. J. Colatsky, A. O. Grant. "What happens when cardiac Na channels lose their function? 1-Numerical studies of the vulnerable period in tissue expressing mutant channels", Cardiovascular Research, Vol. 57, pp. 82-91, 2003. 